\title{
Simulation of Cosmic Rays in the Earth's Atmosphere and Interpretation of Observed Counts in an X-ray Detector at Balloon Altitude Near Tropical Region
}

\author{
Ritabrata Sarkar ${ }^{a, *}$, Abhijit Roy ${ }^{\mathrm{a}}$, Sandip K. Chakrabarti ${ }^{\mathrm{a}}$ \\ ${ }^{a}$ Indian Centre for Space Physics, 43 Chalantika, Garia Station Rd., Kolkata 700084, \\ W.B., India
}

\begin{abstract}
The study of secondary particles produced by the cosmic-ray interaction in the Earth's atmosphere is very crucial as these particles mainly constitute the background counts produced in the high-energy detectors at balloon and satellite altitudes. In the present work, we calculate the abundance of cosmic-ray generated secondary particles at various heights of the atmosphere by means of a Monte Carlo simulation and use this result to understand the background counts in our X-ray observations using balloon-borne instruments operating near the tropical latitude (geomagnetic latitude: $\sim 14.50^{\circ} \mathrm{N}$ ). For this purpose, we consider a 3D description of the atmospheric and geomagnetic field configurations surrounding the Earth, as well as the electromagnetic and nuclear interaction processes using Geant4 simulation toolkit. Subsequently, we use a realistic mass model description of the detector under consideration, to simulate the counts produced in the detector due to secondary cosmic-ray particles.

Keywords: Galactic cosmic rays, Atmospheric background radiation, Balloon-borne X-ray detection
\end{abstract}

\footnotetext{
* Corresponding author

Email addresses: ritabrata.s@gmail.com (Ritabrata Sarkar), aviatphysics@gmail.com (Abhijit Roy), sandipchakrabarti9@gmail.com (Sandip K. Chakrabarti)
} 


\section{Introduction}

Galactic Cosmic Rays (GCRs) are originated from various cosmic sources outside the solar system. They are primarily composed of protons $(\mathrm{H} \sim 89 \%)$, alpha particles $(\mathrm{He} \sim 10 \%)$ and other heavier nuclei. On arriving the Earth's atmosphere they interact with atmospheric nuclei to produce several secondary leptonic and hadronic particles and radiation in the form of a shower. However, before reaching the top of the atmosphere these GCR particles face dynamic electromagnetic environment in the heliosphere which varies with solar activity as well as the geomagnetic field which acts as a shield.

The Coronal Mass Ejection (CME) and solar flares during a period of solar activity affect the space environment in more than one way. A solar activity modulates the flux of the primary GCRs, particularly the low energy part below a few GeV. The Solar Energetic Particles (SEPs) add to the Cosmic Ray (CR) contribution from a few $\mathrm{keV}$ to several $\mathrm{GeV}$. The geomagnetic field deflects GCR particles depending on their rigidity and thus the flux of the low rigidity component gets modified. Intense geomagnetic storms created by the solar activity can affect geomagnetic shielding and thus affect the interplanetary CR distribution (Dorman et al., 1971).

Study of the CR interaction and overall radiation environment in the atmosphere and in space is important for many reasons. The radiation and particles in the overall CR products affect satellite operations (Miroshnichenko, 2003), produce significant background counts in space-based or balloon-borne detectors (Peterson, 1975), affect the crews and passengers in aircrafts (Bütikofer and Flückiger, 2011).

The Monte Carlo (MC) simulation technique has been used in the past to study CR interactions in the atmosphere. For this, Geant4 (Agostinelli et al., 2003) or FLUKA (Battistoni et al., 2007) simulation toolkit is useful. The implementation of microscopic interactions of the CR particles in event by event basis has been done by several others (Zuccon et al., 2003; Heck et al., 1998; Desorgher et al., 2005, 2006; Paschalis et al., 2014). We developed a simulation 
framework using Geant4 to study CR interactions in the atmosphere exploiting full 3D implementation of the atmospheric and geomagnetic structure. We used optimized techniques for efficient simulation and exploited the advantage of deploying the latest models for the atmosphere, magnetosphere and interactions in the process.

Though the implementation of this simulation framework allows us to study detailed radiation environment at various heights in the atmosphere in particular, and in space in general, considering several inputs from GCR or solar CR, here in the present work, we restricted ourselves to use only major components of the GCR (viz. H and He) as the input particles. The GCR contribution from other heavier nuclei has been considered in the He spectrum through a proper scaling factor in the nucleon fraction (Usoskin et al., 2017). In the context of estimating detector background at certain heights and locations, we estimated the spectral and directional distribution of several secondaries generated at that particular locations. However, we also estimate the spectral and directional behaviour at the satellite height and compare the result with the Alpha Magnetic Spectrometer (AMS) result (Alcaraz et al., 2000) for benchmark of the simulation.

The estimation and understanding of the background counts generated by the $\mathrm{CR}$ interactions with the detector and surrounding mass distribution is very important to achieve required sensitivity of the detector, particularly for the high-energy $\gamma$-ray astrophysics where the source intensity could be low. An accurate information of the particle and radiation distributions at the payload environment and proper simulation of the interaction of these particles in the payload from $4 \pi$ solid angle is necessary to develop a proper background rejection algorithm and evaluate the remaining background. There are some models describing the radiation environment which can be used for this purpose (Mizuno et al., 2004; Lei et al., 2006). However, it is more appropriate to generate the desired distributions as a function of energy and observation direction for a given location and a given solar condition. This would be a very ambitious project, however, it is the only way the appropriate corrections can be made. 
In the present work, we consider simulation of CR interactions in the atmosphere with realistic conditions to generate the particle distribution, a potential contributor to the background counts during measurements of extraterrestrial radiation in X-rays during our light-weight paradigm in balloon-borne study (e.g., Chakrabarti et al. (2017); Sarkar et al. (2017) and references therein). We generally use scintillation detectors in these experiments, typically working in the energy range of $\sim 15-100 \mathrm{keV}$. The detector ascends through the atmosphere to reach near $\sim 40-42 \mathrm{~km}$. The estimation of the background counts is important for these detectors like other high-energy astronomical experiments. The major contributors to these experiments are the secondary radiation and particles from the $\mathrm{CR}$ interaction with the atmospheric nuclei. The cosmic gamma-rays also can contribute to the background, depending on the location of the detector. Apart from the direct contribution of these cosmic primary or secondary particles and radiation, there can be a significant background from the induced radioactivity in the detector and surrounding materials due to high energy cosmic-ray particles, along with some natural radioactivity in the detector material (Peterson, 1975). To understand the background counts in the detector, we calculate the radiation distribution from the simulation of CR interaction in the atmosphere. We use this distribution as input to the further simulation to calculate background counts in the detector with proper mass model of the deployed payloads using Geant4 simulation toolkit.

In the following section (Sec. 2), we describe the general simulation procedure and considerations of the CR interactions with the atmosphere and compare the result at satellite heights with AMS measurement (Alcaraz et al., 2000) to validate the simulation. Section 3 gives the particle distribution scenario at balloon altitude, which is used in the next Sec. 4 to evaluate the background counts in a balloon-borne detector. Finally, we discuss the results from this work in Sec. 5 , 


\section{Simulation Procedure for Atmospheric Interactions}

For the simulation of $\mathrm{CR}$ interactions with the Earth atmosphere we need to consider the following aspects. (1) An accurate distribution of the atmospheric molecules inside of which the incident $\mathrm{CR}$ interacts to produce the secondary particle shower. (2) The magnetic field distribution which controls the trajectories of the charged particles also defines the cutoff rigidity for those particles. (3) The spectra of the primary CR components at the top of the atmosphere. (4) The physical processes which describe the interactions of the CR particles with the atmospheric nuclei and atoms. All these simulation aspects have been realized by implementation of a full 3D model of the atmosphere and magnetosphere using Geant4 simulation toolkit with proper distribution of the primary particles and considering suitable interaction processes in Geant4.

\subsection{Atmospheric model}

The distribution of matter in the atmosphere depends on the location, time and other conditions, such as, solar activity. This leads to different interaction probabilities of the particles and radiation varying dynamically with location and time. We considered the NRLMSISE-00 standard atmospheric model (Picone et al., 2002) up to $100 \mathrm{~km}$ from the Earth surface with proper input parameters for location, time and solar condition (parameterized by solar 10.7 $\mathrm{cm}$ radio flux and magnetic $\mathrm{A}_{p}$ index) to describe the atmosphere in the simulation. The location and time used for the simulation was chosen to match with the experimental conditions we conducted. The solar $10.7 \mathrm{~cm}$ flux and $\mathrm{A}_{p}$ index value were fixed, depending on the time of the experiment, at $100 \times$ $10^{-22}$ watt $/ \mathrm{m}^{2} / \mathrm{Hz}$ and 5 respectively for the simulation (NOAA, 2018). We have limited the atmospheric height till $100 \mathrm{~km}$ considering the fact that most of the high-energy CR particles interact in the atmosphere below this altitude. This consideration reduces the simulation load because the step size of the particle tracks are relatively smaller in the atmospheric medium compared to other regions without any matter distribution. The whole atmosphere is subdivided 
into 100 concentric spherical layers of equal logarithmic altitude (in $\mathrm{km}$ ). We calculated the temperature, pressure, total mass density and number density of each of the major molecular components (e.g. $\mathrm{N}_{2}, \mathrm{O}_{2}, \mathrm{He}, \mathrm{Ar}, \mathrm{H}, \mathrm{N}, \mathrm{O}$ ) in the atmospheric layers from the existing model to construct the whole atmosphere.

\subsection{Geomagnetic model}

The magnetic field surrounding the Earth affects both the primary and secondary CR charged particles and subsequently the generation of neutral particles through deviation and entrapment resulting in the rigidity cutoff of incoming charged particles. It also defines the trajectories of all the charged particles. The field distribution is highly dynamic and strongly coupled to the solar activity in addition to the relatively slower secular variation in the geomagnetic field. The magnetic field in the vicinity of the Earth has two components: (i) the inner magnetic field due to the Earth's magnetism near the Earth surface which extends up to about 4 Earth radii from Earth surface and (ii) the external magnetic field which depends on the Interplanetary Magnetic Field (IMF) and solar conditions. We consider the magnetic field distribution up to 25 Earth radii. The inner magnetic field used in this simulation is calculated using the 12th generation IGRF model (Thébault et al., 2015) with proper input parameters. The external magnetic field is calculated using Tsyganenko Model (Tsyganenko and Andreeva, 2016). For our simulation purpose we considered solar parameters, required in the magnetospheric models during the time of 92nd Dignity mission (Chakrabarti et al., 2017) of Indian Centre for Space Physics (ICSP). We will use the simulation results to compare and understand the data obtained from this mission. The date and time of interest is: 11th May, 2016 at around 5:35 UT. During the simulation of the charged particle tracks in Geant4, the resulting magnetic fields are calculated at the position of each step of the particle track and the track is recalculated integrating the equation of motion of the charged particle in the field. We used G4CashKarpRKF 45 stepper method (Geant4, 2019a) provided in Geant4 for the integration purpose. 


\subsection{Primary particle generation}

In the context of the present work, where we study the interaction of the cosmogenic particles to produce secondary particles in the atmosphere, we only consider the GCR. The most abundant components of GCR are the protons $(\mathrm{H})$ and helium nuclei (He) which constitute about $99 \%$ of the GCR flux. So we consider these two species of the CR particles along with the Cosmic Diffuse Gamma-Ray Background (CDGRB) (Dean et al., 1991) as the main inputs of our simulation in order to evaluate the cosmogenic background of the X-ray detector. Here in this work, we are not considering the primary CR electrons because our present study is confined in the relatively low latitude where the cut-off energy is high and also the trigger efficiency in the detector under consideration for electrons is very low.

The primary radiation and neutral particles reach the Earth undeviated and interact with the atmosphere, while the charged particles are deflected by the Earth's magnetic field. In the ideal case, we need to generate an isotropic distribution of particle flux at a great distance where the flux distortion due to magnetic field is negligible and then calculate the particle flux at the desired location (say, at the top of the atmosphere). This is clearly an inefficient process for the simulation. Instead, we follow the prescription for the backtracing method as considered by Zuccon et al. (2003) and in PLANETOCOSMICS (Desorgher et al., 2006). We produce an isotropic distribution of the charged particles from a geocentric spherical surface at $500 \mathrm{~km}$ above the Earth surface and backtracked them to reach an outer surface at 25 Earth radii in presence of the magnetic field distribution described in Sec. 2.2. We select only those particles which reach the outer sphere as the allowed GCR particle tracks and proceed with the simulation for atmospheric interactions.

Based on the direct GCR observations in the very local intersteller medium by Voyager 1 (Stone et al., 2013) after it crossed the heliopause and using the PAMELA (Adriani et al., 2013) and AMS02 (Aguilar et al., 2015) data, new model to represent the very local interstellar spectrum (LIS) has been proposed by Vos and Potgieter (2015). In this work we used the same LIS represented 
by:

$$
J_{L I S}\left(E_{k}\right)=N \frac{E_{k}^{1.12}}{\beta^{2}}\left(\frac{E_{k}+0.67}{1.67}\right)^{-\alpha},
$$

where, $E_{k}$ is the kinetic energy of the particles, $N$ - normalization factor, $\alpha$ index of the power-law. The modified differential particle flux at $1 \mathrm{AU}$ is given by (Herbst et al., 2017):

$$
J\left(E_{k}, \phi\right)=J_{L I S}\left(E_{k}+\Phi\right) \frac{E_{k}\left(E_{k}+2 M c^{2}\right)}{\left(E_{k}+\Phi\right)\left(E_{k}+\Phi+2 M c^{2}\right)},
$$

where, $M$ - particle mass, $c$ - speed of light and $\Phi=(Z / A) \phi . Z$ and $A$ - atomic and mass number of the particle and $\phi$ - solar modulation parameter. To produce the primary $\mathrm{H}$ flux we considered $A=2.70 \times 10^{3}$ particles $s^{-1} m^{-2} \mathrm{sr}^{-1} \mathrm{GeV}^{-1}$ and $\alpha=3.93$. The solar modulation parameter $\phi$ is fixed at $524 \mathrm{MV}$ (Usoskin et al., 2017) for the time considered during the simulation for comparison with the balloon-borne experiment while $\phi=555 \mathrm{MV}$ was considered for the comparison with AMS result in Sec. 2.5. For the He spectrum we use $\alpha=3.89$, which is required to fit the He spectrum given in Bisschoff and Potgieter (2016). The normalization factor is modified using the nucleon fraction for He particles with an additional factor of 0.3 which also includes the contribution from other heavier species in GCR (Usoskin et al., 2017).

Apart from the solar modulation, a more prominent cause of modulation of the primary flux comes from the rigidity cutoff due to geomagnetic field when the charged particles approach the Earth. This modulation is achieved in the simulation through the incorporation of the accurate geomagnetic field configuration. The generated primary flux of the $\mathrm{H}$ and $\mathrm{He}$ and modulation due to the geomagnetic cutoff is shown in Fig. 1. This is the average flux over the geomagnetic latitude region of $0-57.3^{\circ}$. Here the rigidity cutoff of the charged particles is manifested from the simulation inherently due to the interaction of the individual particle tracks with the defined geomagnetic field and there is no need to separately supply the rigidity cutoff value to modify Eqn. 2 ,

Considering the geomagnetic cutoff effect at low energy and decreased particle flux at high energy due to power-law distribution, we restricted the primary generation in the energy range of $0.1-800.0 \mathrm{GeV} / \mathrm{n}$. The particles in this energy 

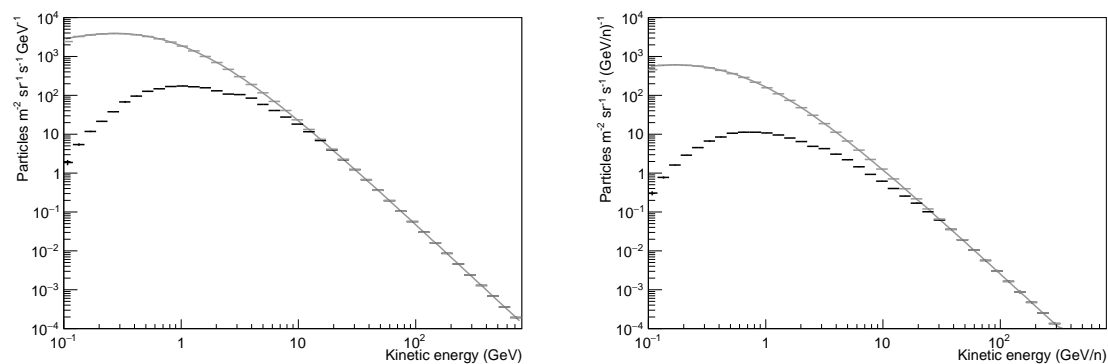

Figure 1: Primary particle flux considered in the simulation: (left) H flux; (right) He flux. Gray data points represent original primary flux generated according to Eqn. 2 Black points represent the same fluxes after the geomagnetic cutoff (see text for details).

range carry $\sim 98 \%$ of the total energy of CR particles allowing us to include most of the energy transport interactions in our simulation (Zuccon, 2002). To maintain the statistical significance of counts in the whole energy range, we subdivided the whole energy range in 5 sub-ranges (0.1-0.3, 0.3-3.0, 3.0-30.0, 30.0-170.0 and 170.0-800.0 GeV/n). We simulated a total of $3.5 \times 10^{5}$ particles both for $\mathrm{H}$ and $\mathrm{He}$ and calculated the particle abundance to be simulated in each energy range, keeping in mind the number of secondaries produced in the energy range. This is done so that the statistical significance of the secondary counts in each range remains comparable.

Because of our particular interest in relatively low energy photons, we also simulated CDGRB photons in the energy range of $10 \mathrm{keV}-0.1 \mathrm{GeV}$. For this purpose, we used the primary photon spectrum observed by Swift BAT (Ajello et al., 2008) for energies $\leq 1 \mathrm{MeV}$ and for higher energies up to $0.1 \mathrm{GeV}$ we considered COMPTEL observation (Weidenspointner, 1999):

$$
J(E)= \begin{cases}\frac{N_{1}}{\left(\frac{E}{E_{b}}\right)^{\alpha_{1}}+\left(\frac{E}{E_{b}}\right)^{\alpha_{2}}}, & \text { for } \mathrm{E} \leq 1 \mathrm{MeV}, \\ N_{2}\left(\frac{E}{5 M e V}\right)^{-\alpha_{3}}, & \text { for } \mathrm{E}>1 \mathrm{MeV},\end{cases}
$$

where, $N_{1}=1.015 \times 10^{9}, N_{2}=1.12 \times 10^{3}$ photons $s^{-1} m^{-2} s^{-1} G e V^{-1}, \alpha_{1}=$ $1.32, \alpha_{2}=2.88, \alpha_{3}=2.2$ and $E_{b}=29.99 \mathrm{keV}$. The whole energy range is divided into 4 sub-ranges $\left(10-100,100-10^{3}, 10^{3}-10^{4}\right.$ and $\left.10^{4}-10^{5} \mathrm{keV}\right)$ and simulated $10^{5}$ 
photons in each energy sub-range to maintain statistical significance of counts.

\subsection{Physical interaction models}

To simulate interaction of high energy particles with the atmosphere we need an optimal interaction model. We used the reference physics list: QGSP physics list with Binary Cascade model QGSP_BIC_HP (Geant4, 2019b), provided in Geant4 which is recommended for cosmic-ray applications and optimally covers the interaction processes relevant here. In this current simulation procedure, the relevant energy interval is $10 \mathrm{keV}-800 \mathrm{GeV}$. The hadronic interactions considered in the physics list are recommended to be suitable at least up to $10 \mathrm{TeV}$, while the low energy limit mentioned to be valid from 0 . To handle the electromagnetic part this model this physics list use the "standard" Geant4 electromagnetic physics which is valid from $100 \mathrm{eV}$ to $100 \mathrm{TeV}$. This physics list includes the high precision neutron interaction model to handle the elastic and inelastic scattering, capture and fission more accurately from $20 \mathrm{MeV}$ down to thermal neutrons.

\subsection{Validation of simulation using AMS data}

To validate the simulation procedure by comparing the results with the AMS

proton flux measurement (Alcaraz et al., 2000), we calculated the total proton flux (from primary CR and secondary generated) at the satellite altitude of $\sim$ $400 \mathrm{~km}$ and within $32^{\circ}$ of AMS z-direction (here we consider the zenith and nadir directions respectively for downward and upward particles). The simulation input parameters for the atmospheric and magnetic field models were adjusted according to the time of the AMS observation used here for comparison. We considered the calculations in the corrected geomagnetic latitude $\left(\theta_{M}\right.$, as mentioned in Alcaraz et al. (2000)) range of $40.1^{\circ}<\theta_{M}<45.8^{\circ}$. In Fig. 2 we show the downward and upward proton fluxes calculated from the simulation. The corresponding AMS measured fluxes are presented in the same plot for comparison. We also conducted a goodness-of-fit test to quantify the agreement of the results by using Pearson's $\chi^{2}$ test. For the downward protons this 

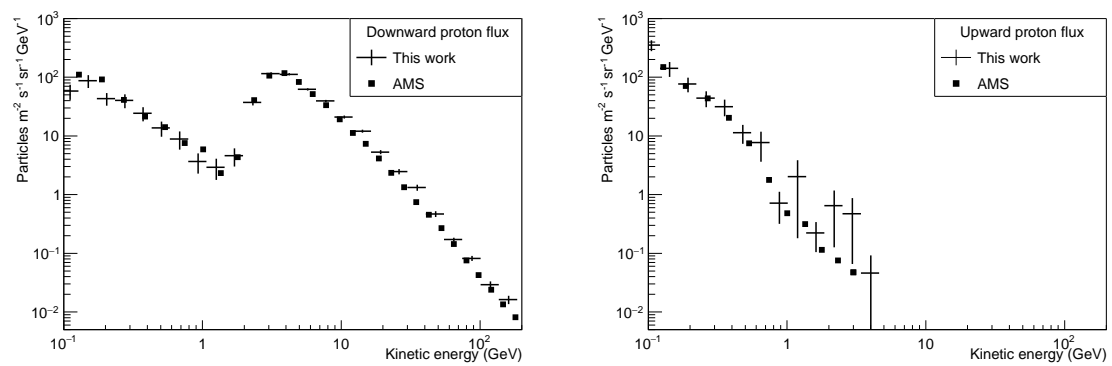

Figure 2: Downward/upward proton flux at the satellite height of $400 \mathrm{~km}$ due to $\mathrm{H}$ and $\mathrm{He}$ particle interactions in the latitude range of $40.1^{\circ}<\theta_{M}<45.8^{\circ}$ and their comparison with AMS data.

test yielded a $\chi^{2} / \mathrm{NDF}$ value $16.44 / 18$ with probability factor 0.56 and for the upward proton we found the respective values to be $12.93 / 11$ and 0.30 . Thus the calculated results are in general agreement with the AMS results.

\section{Particles at Balloon Height}

To understand the background counts in a detector at the balloon altitude we calculated the flux distribution of different CR generated secondary particles such as: protons, neutrons, electrons, positrons, muons (+/-) and photons at an altitude of $\sim 30 \mathrm{~km}$ (atmospheric depth $\sim 11.52 \mathrm{~g} / \mathrm{cm}^{2}$ ). Depending on the mass distribution model of the payloads, these particles may interact and produce counts in the detectors.

The spectral distribution of the particles at the balloon altitude and at geomagnetic latitude of $11.5^{\circ}<\theta_{M}<17.2^{\circ}$ are shown in Fig. 3. The choice of this particular latitude is to calculate and compare the background of a balloon borne experiment near the tropic of cancer which is discussed in more detail in Sec. 4. Here we considered to calculate the particle distributions within whole $90^{\circ}$ angle from zenith and nadir for downward and upward particles respectively. This has been done to include the effect of directional distribution of the particles interacting with the payload mass.

The secondary particles at this height is much more abundant than the 
primaries as compared to those at the satellite height. Though, the downward and upward proton fluxes differ with diminishing contribution of the upward protons, the upward and downward neutron and photon fluxes are comparable at least at the lower energy domain. Similar behavior, to some extent, can be seen for the $\mathrm{e}^{-} / \mathrm{e}^{+}$which (generated from CR interactions in the atmosphere) are scarce at the satellite height. However for the muon component, the downward counterparts are more prominent.

\section{Comparison with Observed Data}

We used energetic and directional distribution of different particles at the balloon altitude obtained from the simulation described in Sec. 3, as the input for the simulation to estimate the counts due to these particles in a balloon-borne detector. This detector was deployed in an experiment designed to measure extraterrestrial radiation from astronomical sources. In this case, the secondary and primary $\mathrm{CR}$ particles at balloon altitude interacts with the payload mass, as well as with the active detector volume to generate detector counts which contribute to the detector background. Estimation of the background and its reduction is very crucial for these detectors as they deal with relatively low source counts.

\subsection{Experiment overview}

Indian Centre for Space Physics has been measuring CR induced radiations in the atmosphere and high-energy X-rays from astrophysical sources for over a decade through its low-cost, near-space program with rubber or polystyrene meteorological balloons. Here, light-weight payloads are used without any pointing device or heavy shielding which make the data reduction tedious in the absence of an accurate model of background radiation. The details of the program can be found in Chakrabarti et al. (2014, 2015, 2017). In this paper, we concentrate on the results obtained in Dignity 92 mission. Our scientific payload comprised of a phoswich scintillator detector which consists of a combination of $3 \mathrm{~mm}$ thick 

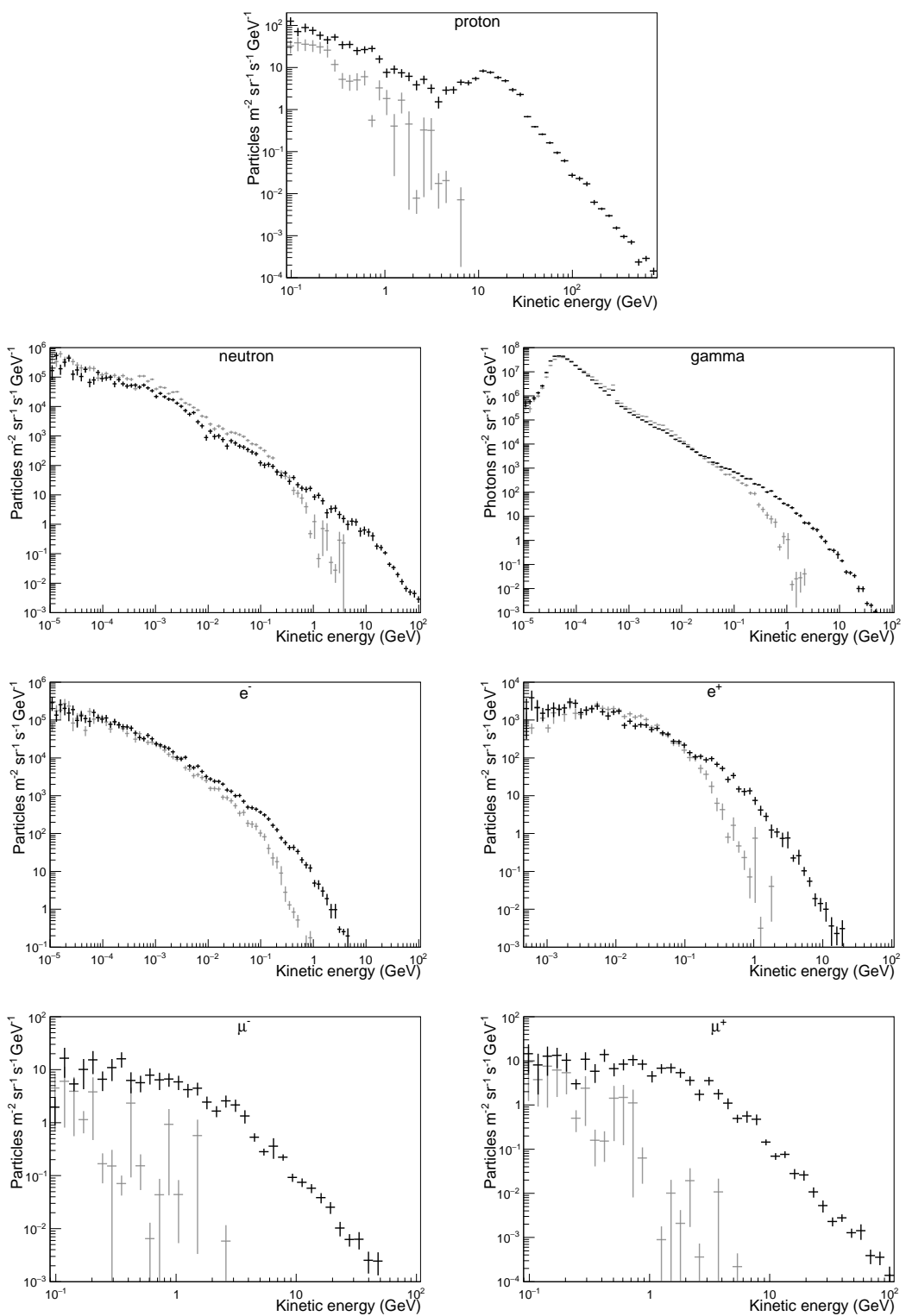

Figure 3: Downward (black)/upward (gray) particle flux for proton, neutron, photon, $e^{-}$, $e^{+}, \mu^{-}$and $\mu^{+}$at balloon altitude of $30 \mathrm{~km}\left(\sim 11.52 \mathrm{~g} / \mathrm{cm}^{2}\right)$ and in the latitude range of $11.5^{\circ}<\theta_{M}<17.2^{\circ}$ due to cosmic ray $\mathrm{H}$, He and CDGRB photon interactions considering whole $90^{\circ}$ angle from zenith/nadir. 
$\mathrm{NaI}(\mathrm{Tl})$ and $25 \mathrm{~mm}$ thick $\mathrm{CsI}(\mathrm{Na})$ crystal discs of $116 \mathrm{~mm}$ diameter mounted on a Photo-Multiplier Tube (PMT) housed in an aluminum casing. The detector is fitted with a collimator (made of $0.5 \mathrm{~mm}$ thick lead) to reduce the off-axis external background counts. The detector works in the energy range of $\sim 15-100$ $\mathrm{keV}$ and counts in the detector are triggered by the energy depositions in the scintillator crystals. However, the detector is operated in anticoincidence mode to record only exclusive full energy deposition by an event (photon) in the NaI crystal. The detector counts are recorded in the on-board data storage system during the mission flight with the help of suitable readout and data processing system. There are other ancillary instruments to accomplish the mission. More detail about the detector and the payload may be found in Chakrabarti et al. (2017); Sarkar (2018).

In the mission under consideration, the payload was launched from Muluk, West Bengal, India (latitude: $23.64^{\circ} \mathrm{N}$; longitude: $87.71^{\circ} \mathrm{E}$; geomagnetic latitude: $14.5^{\circ} \mathrm{N}$ ) on May 11, 2016 at 03:42 UT. The payload went up to $\sim 41$ $\mathrm{km}\left(\sim 2.03 \mathrm{~g} / \mathrm{cm}^{2}\right)$, however, for the sake of general comparison and understanding of the experimental data using our simulated result, we considered the experimental data at $30 \mathrm{~km}\left(11.52 \mathrm{~g} / \mathrm{cm}^{2}\right)$ during the ascend of the payload.

We performed a detailed simulation of the detector to calculate the background counts due to the $\mathrm{CR}$ generated particles and radiations using the Geant4 simulation framework. A proper mass model of the payload is crucial for these types of simulation as the materials used in the payload construction may produce significant radiation counts which add to the detector background. However, due to the unique payload design of the mission under consideration where the payload casing is mainly made up of styrofoam, the mass distribution of the payload frame and other ancillary instruments can be neglected. The electronic circuits and other ancillary instruments are very small in volume and weight and are not considered in the simulation. The mass model of the detector considered for the simulation is shown in Fig. 4. 


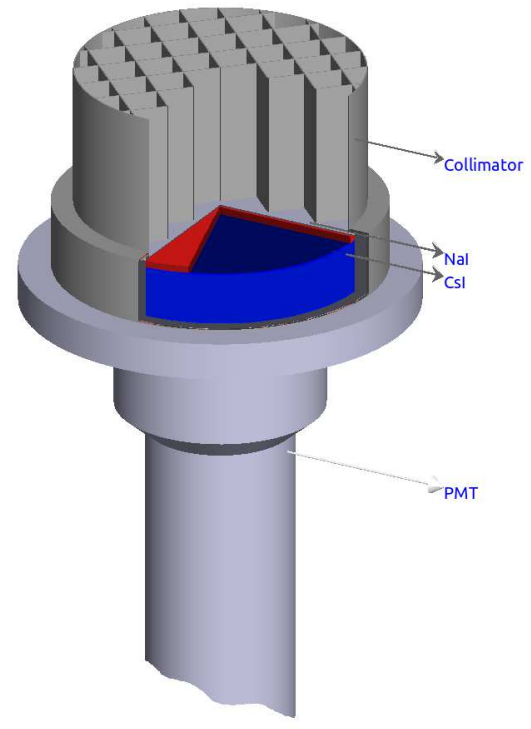

Figure 4: Mass model of the phoswich detector used for the background simulation.

\subsection{Detector background calculation}

There are several contributors to the background counts for the astronomical detectors on board balloons or satellite platforms. The contributions may be from the external sources such as: CR generated particles, trapped particles, CDGRB radiation etc. or due to the radiations internal to the detector, such as, induced radioactivity from the activated isotopes, induced spallation radiation in the active detector, natural radioactivity from the detector or surrounding materials etc. (Peterson, 1975).

Here, in this work we have explicitly calculated the contribution to the detector background due to cosmogenic particles and radiation. For this purpose we used the information of the simulated production of secondary CR particles along with the CDGRB contribution as the input to the simulation of the detector background. We used the spectral information of the secondary particles in the energy range which is able to generate significant number of triggers in the detector. We also took into account, the zenith angle distribution of the particles and radiation during the generation of the input particles for the sim- 


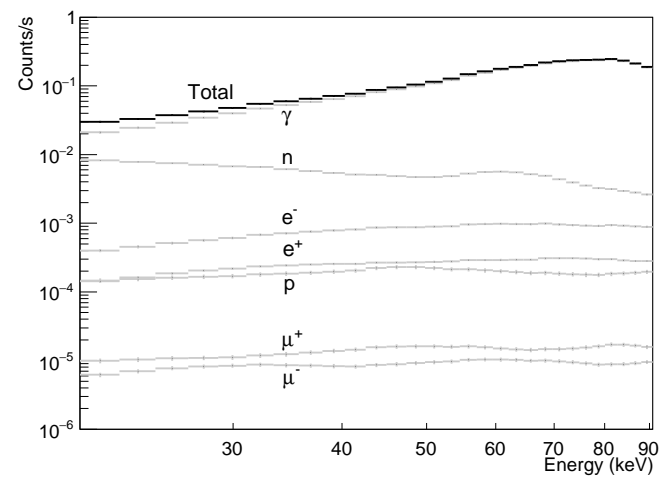

Figure 5: Contribution to the detector counts due to different cosmogenic radiation and particles. The total CR contribution is shown in black while the individual components are plotted in gray.

ulation. However, due to the axial symmetry of the detector along the zenith direction we did not consider the azimuthal distribution of the particle generation. Digitization of the energy deposition in the NaI crystal was thoroughly done by incorporating the anti-coincidence effects with CsI crystal and response function of the detector obtained during its calibration to produce the background counts in the detector (Sarkar et al., 2019). The contribution of several cosmogenic particles and radiation produced by the $\mathrm{CR}$ interaction in the atmosphere along with the CDGRB photons, to the detector background counts is shown in Fig. 5. Our result shows that the major contributors to the detector background are photons $(\sim 95 \%)$ and neutrons $(\sim 4 \%)$ of the secondary CR.

However, the external background from the CR origin comprises only a part of the total background of the detector, in the energy range under consideration, as it is evident from Fig. 6. The lower energy part is dominated by the internal background of the detector by the induced radioactivity due to activation by capture of neutrons in the detector or/and shielding material or by spallation radioactivity due to breakup of high $\mathrm{Z}$ target nucleus in the scintillator crystal. This cosmogenic induced background depends on the position of the payload in the atmosphere (Sarkar et al., 2019), as the secondary cosmic ray flux varies 
with the altitude and location in the atmosphere. For instance, the observed count rate by the detector near $20 \mathrm{keV}$ (where internal background is dominating) can be approximated by the exponential form: $\exp \left(0.116+0.013 \times D_{a t m}\right)$, where $D_{a t m}$ is the atmospheric depth at different altitude (in the range 20 - 40 $\mathrm{km}$ or $\left.55.77-2.38 \mathrm{~g} / \mathrm{cm}^{2}\right)$.

The induced background spectrum produced in the detector depends on several factors: the CR particle flux inducing the radioactivity in the detector and surrounding materials, production rate of several radioactive isotopes, decay of the radioactive isotopes in the allowable observation time and efficiency of detection of the decay counts in the detector (Fishman, 1972). The final background spectrum due to these induced activity composed of several decay lines and continua from beta decay. Here, in this work we have not calculated the induced background from the radioactive products in detail. Instead, to explain the total background counts in the detector, we have empirically fitted the induced background part using four components. The lower energy part has been treated with an exponential cutoff of the form:

$$
\frac{d F}{d E}=\frac{F_{c}}{E_{f}} \exp \left(-\frac{E}{E_{f}}\right)
$$

as described by Fishman (1972). Here, $F_{c}$ represents the rate of detected counts and $E_{f}$ is the e-folding energy. The value of $F_{c}$ depends on the incident $\mathrm{CR}$ and hence on the location of the detector. We also needed to use three other Gaussian lines due to radiation from the induced or natural radioactive isotopes ${ }^{210} \mathrm{~Pb}$ at $\sim 44 \mathrm{keV},{ }^{125} \mathrm{I}$ at $67.2 \mathrm{keV}$ (Adhikari et al., 2017) and ${ }^{127} \mathrm{I}$ at $57 \mathrm{keV}$ (Peterson, 1975). The spread of these Gaussian lines was fixed to the resolution of the detector at the corresponding energies but their normalization factors were free for the fit. For the background spectrum of the detector at the location under consideration, the fit gives $F_{c}=77.79$ counts $/ s, E_{f}=$ $13.29 \mathrm{keV}$ and three normalization factors as $0.046,0.089$ and 0.052 counts/s. The internal background along with the external CR background explains the detector background satisfactorily. The observed background spectrum along with the external CR contribution calculated from the simulation and fitted 


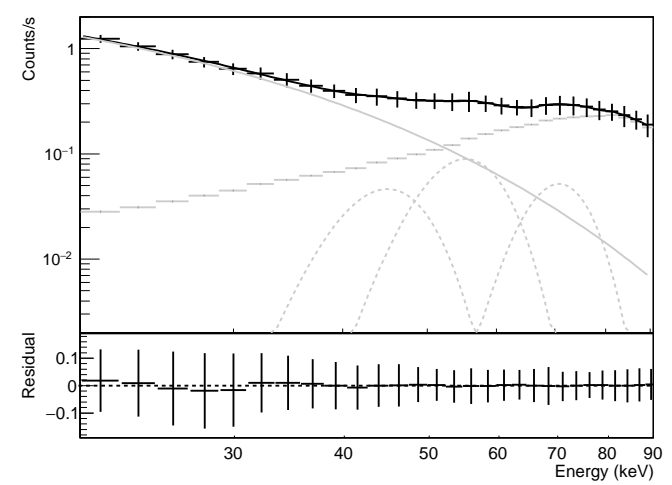

Figure 6: Components of different background contributors to the total background of the detector at $30 \mathrm{~km}$ altitude. The total detected background is given by black points; external CR contribution calculated from the simulation given by gray points, low-energy exponential form of the induced internal radioactivity given by gray solid line with three other Gaussian (dashed gray lines); the total calculated background is shown by black solid line along the detector data points. The lower panel shows the residual to the fitting.

background components are shown in Fig. 6. The lower panel of the Figure shows the residual values. The total background count rate at $30 \mathrm{~km}$ altitude in the energy range of $20-90 \mathrm{keV}$ is 12.28 counts/s. This counting rate varies with the atmospheric depth $\left(D_{a t m}\right)$ roughly as the exponential form: $\exp \left(2.34+0.0148 \times D_{a t m}\right)$. Thus, for example, the expected count rate at the height of $40 \mathrm{~km}\left(D_{\text {atm }}=2.38 \mathrm{~g} / \mathrm{cm}^{2}\right)$ is approximately $10.8 \mathrm{counts} / \mathrm{s}$ in the same energy range.

\section{Conclusions}

Along with several other important implications, the interaction of Galactic cosmic rays in the atmosphere plays a crucial role in the astronomical experiments, particularly for the balloon borne detectors. The background counts produced in the detectors by these CR generated particles limit the detectibility of the astrophysical sources and demand a thorough study. Here in this work, in the context of a light-weight balloon-borne experiment to study extraterrestrial radiation sources, we studied the CR generated particles in the 
atmosphere and their effects on the detector. In this course of work, we developed a simulation framework using Geant4 toolkit to study the interaction of the extraterrestrial radiation and particles with Earth atmosphere in presence of the geomagnetic field. We validated the simulation results at satellite height using the AMS result. Then, we calculated the spectral and angular distribution of various secondary particles at the balloon height due to the interaction of $\mathrm{H}$ and $\mathrm{He}$ (effectively including the contribution from heavier nuclei) which are the most abundant species of cosmic rays. Although, we considered only the interaction of the GCR with the atmosphere, this simulation framework can be extended to study other interactions, such as, the effects of SEP in our atmosphere.

We used the results produced by the CR interaction with the atmosphere to estimate the background counts in a balloon borne astronomical detector. However, the external CRs produce only a part of the detector background in its operating energy range and we also need to consider the internal background induced by the CRs or energetic particles of other origins. Here, we have treated the problem of internal background only empirically which can be calculated more extensively using detailed analytical (Dvre and Morfill , 1971) and simulation (Adhikari et al., 2017) procedures.

\section{Acknowledgments}

The authors would like to thank the ICSP balloon team members, namely, Mr. D. Bhowmick, Mr. A Bhattacharya, Mr. S. Midya, Mr. H. Roy, Mr. R. C. Das and Mr. U. Sardar for their valuable supports in various forms during the mission operations and data collection. This work been done under partial financial support from the Science and Engineering Research Board (SERB, Department of Science and Technology, Government of India) project no. EMR/2016/003870. We also thank the Higher Education department for a Grant-In-Aid which allowed us to carry out the research activities at ICSP. All the data shown in this work are available from the authors. 


\section{References}

Adhikari G., Adhikari P., Ha C., 2017, Understanding NaI(Tl) crystal background for dark matter searches, Eur. Phy. J. C., 77, 9, doi:10.1140/epjc/s10052-017-5011-6

Adriani O., Barbarino G. C., Bazilevskaya G. A., et al., 2013, Time dependence of the proton flux measured by pamela during the 2006 July 2009 December solar minimum, ApJ, 765, 91, doi:10.1088/0004-637x/765/2/91

Agostinelli, S., Allison, J., Amako, K. et al., 2003, Geant4 - a simulation toolkit, Nucl. Instr. Methods A, 506/3, 250-303, doi:10.1016/S0168-9002(03)01368-8

Aguilar, M., Aisa, D., Alpat, B. et al., 2015, Precision Measurement of the Proton Flux in Primary Cosmic Rays from Rigidity $1 \mathrm{GV}$ to $1.8 \mathrm{TV}$ with the Alpha Magnetic Spectrometer on the International Space Station, PhRvL, 114, 171103, doi:10.1103/PhysRevLett.114.171103

Ajello, M., Greiner, J., Sato, G., et al., 2008, Cosmic X-ray background and Earth albedo spectra with Swift BAT, ApJ, 689, 666-677, doi:10.1086/592595

Alcaraz, J., Alvisi, D., Alpat, B. et al., 2000, Protons in near Earth orbit, PhLB, 472, 215-226, doi:10.1016/S0370-2693(99)01427-6

Battistoni, G., Cerutti, F., Fassò, A. et al., 2007, The FLUKA code: description and benchmarking, Albrow, M., Raja, R. (Eds.), Hadronic Shower Simulation Workshop. Proceedings held at Batavia, Illinois, USA, American Institute of Physics, AIP Conference Proceedings, Melville, NY, pp. 31-49, doi:10.1063/1.2720455

Bisschoff, D., Potgieter, M. S., 2016, New local interstellar spectra for protons, helium and carbon derived from PAMELA and Voyager 1 observations, Astrophys Space Sci, 361, 48, doi:10.1007/s10509-015-2633-8

Bütikofer, R., Flückiger, E. O., 2011, Radiation doses along selected flight profiles during two extreme solar cosmic ray events, ASTRA, 7/2, 105-109, doi:10.5194/astra-7-105-2011 
Chakrabarti, S. K., Bhowmick, D., Chakraborty, S., Palit, S., Mondal, S. K., Bhattacharya, A., Midya, S., Chakrabarti, S., 2014, Study of properties of cosmic rays and solar X-ray flares by balloon borne experiments, IJP, 88, 333-341. doi:1007/s12648-13-0424-z

Chakrabarti, S. K., Bhowmick, D., Sarkar, R., Bhattacharyya, A., Midya, S., 2015, Unique high energy experiment initiative by ICSP with weather balloons, Ouwehand, L. (Ed.), Proceedings of 22nd ESA Symposium on European Rocket and Balloon Programmes and Related Research, Troms $\varnothing$, Norway, ESA SP-730, 557.

Chakrabarti, S. K., Sarkar, R., Bhowmick, D., Bhattacharya, A., 2017, Study of high energy phenomena from near space using low-cost meteorological balloons, Exp. Astron., 43(3), 311-338. doi:10.1007/s10686-017-9540-7

Dean, A. J., Lei, F., Knight, P. J., 1991, Background in space-borne low-energy gamma-ray telescopes, Space Science Reviews, 57, 109-186, doi:10.1007/BF00195952

Desorgher, L., Flückiger, E. O., Gurtner, M., et al., 2005, Atmocosmics:. a Geant4 Code for Computing the Interaction of Cosmic Rays with the Earth's Atmosphere, IJMPA, 20/29, 6802-6804, doi:10.1142/S0217751X05030132

Desorgher, L., Flückiger, E. O., Gurtner, M., 2006, The PLANETOCOSMICS Geant4 application, 36th COSPAR Scientific Assembly, meeting abstract from the CDROM, \#2361.

Dorman, L. I., Smirnov, V. S., Tyasto, M. I., 1971, Cosmic rays in the Earth's magnetic field, Nauka, Moscow.

Dyre, C. S., Morfill, G. E., 1971, Contribution to the Background Rate of a Satellite X-Ray Detector by Spallation Products in a Caesium Iodide Crystal, Ap\&SS, 14, 243-258, doi:10.1007/BF00649205

Fishman, G. J., 1972, Cosmic-ray effects on diffuse Gamma-ray measurements, ApJ, 171, 163-167, doi:10.1086/151267 
Geant4, 2019a, Book for application developers, https://geant4-userdoc.web.cern.ch/geant4-userdoc/UsersGuides/ForApplicationDeveloper/html/Detector/electroMagneticField.html, Accessed: 30 August, 2019.

Geant4, 2019b, Physics List Guide, http://geant4-userdoc.web.cern.ch/geant4userdoc/UsersGuides/PhysicsListGuide/html/reference_PL/QGSP_BIC.html, Accessed: 02 September, 2019.

Heck, D., Knapp, J., Capdevielle, J. N., 1998, CORSIKA: a Monte Carlo code to simulate extensive air showers, Technical Report FZKA 6019, Forschungszentrum Karlsruhe GmbH, Karlsruhe (Germany), TIB Hannover, D-30167 Hannover (Germany), https://web.ikp.kit.edu/corsika/physics_description/corsika_phys.pdf

Herbst, K., Muscheler, R., Heber, B., 2017, The new local interstellar spectra and their influence on the production rates of the cosmogenic radionuclides ${ }^{10} \mathrm{Be}$ and ${ }^{14} \mathrm{C}$, J. Geophys. Res. Space Phys., 122, 23-34, doi:10.1002/2016JA023207

Lei, F., Hands, A., Clucas, S., et al., 2006, Improvement to and Validations of the QinetiQ Atmospheric Radiation Model (QARM), IEEE Trans. on Nuc. Sc., 53, 1851-1858, doi:10.1109/tns.2006.880567

Miroshnichenko, L. I., 2003, Radiation hazard in space, Springer Science + Business Media, doi:10.1007/978-94-017-0301-7

Mizuno, T., Kamae, T., Godfrey, G., et al., 2004, Cosmic-ray Background Flux Model Based on a Gamma-Ray Large Area Space Telescope Balloon Flight Engineering Model, ApJ, 614/2, 1113-1123, doi:10.1086/423801

Paschalis, P., Mavromichalaki, H., Dorman, L. I. et al., 2014, Geant4 software application for the simulation of cosmic ray showers in the Earth's atmosphere, New Astron., 33, 26-37, doi:10.1016/j.newast.2014.04.009 
Peterson, L. E., 1975, Instrumental technique in X-ray astronomy, Ann. Rev. Astron. Astrophys., 13, 423-509, doi:10.1146/annurev.aa.13.090175.002231

Picone, J. M., Hedin, A. E., Drob, D. P., Aikin, A. C., 2002, NRL-MSISE-00 Empirical Model of the Atmosphere: Statistical Comparisons and Scientific Issues, J. Geophys. Res. Space Phys., 107, 1468, doi:10.1029/2002JA009430

Sarkar, R., Chakrabarti, S. K., Pal, P. S., Bhowmick, D., Bhattacharyya, A., 2017, Measurement of secondary cosmic ray intensity at Regener-Pfotzer height using low-cost weather balloons and its correlation with solar activity, AdSpRes, 60, 991-998, doi:10.1016/j.asr.2017.05.014

Sarkar, R., 2018, Detector Development and Optimization for Space Based Astronomy from Satellites and Balloons, in : Mukhopadhyay, B., Sasmal, S. (Eds.), in Exploring the Universe: From Near Space to Extra-Galactic, Springer International Publishing AG, part of Springer Nature, 53, 29, 371385, doi:10.1007/978-3-319-94607-8_29

Sarkar, R., Chakrabarti, S. K., Bhowmick, D., Bhattacharyya, A., Roy, A., 2019, Detection of Crab radiation with a meteorological balloon borne phoswich detector, Exp. Astron., 47, 345-358, doi:10.1007/s10686-019-09632-0

NOAA, 2018, National Oceanic and Atmospheric Administration. https://www.ngdc.noaa.gov/stp/geomag/kp_ap.html, Accessed: 10 November, 2018.

Stone, E. C., Cummings, A. C., McDonald, F. B., et al., 2013, Voyager 1 Observes Low-Energy Galactic Cosmic Rays in a Region Depleted of Heliospheric Ions, Science, 341, 150-153, doi:10.1126/science.1236408

Thébault, E., Finley, CC., Toh, H., 2015, Special issue "International Geomagnetic Reference Field: the 12th generation", Earth Planets and Space, 67, 79-98, doi:10.1186/s40623-015-0313-0 
Tsyganenko, N. A., Andreeva, V. A., 2016, An empirical RBF model of the magnetosphere parameterized by interplanetary and ground-based drivers, J. Geophys. Res. Space Phys., 121, 10786-10802, doi:10.1002/2016JA023217

Usoskin, I. G., Gil, A., Kovaltsov, G. A.et al., 2017, Heliospheric modulation of cosmic rays during the neutron monitor era: Calibration using PAMELA data for 2006-2010, J. Geophys. Res. Space Phys., 122, 3875-3887, doi:10.1002/2016JA023819

Vos, E. E., Potgieter, M. S., 2015, New modeling of galactic proton modulation during the minimum of solar cycle 23/24, ApJ, 815, 119, doi:10.1088/0004$637 \mathrm{X} / 815 / 2 / 119$

Weidenspointner, G., 1999, The Origin of the Cosmic GammaRay Background in the COMPTEL Energy Range, Ph.D. thesis, Max-Planck-Institut für extraterrestrische physik, https://mediatum.ub.tum.de/doc/602832/602832.pdf

Zuccon, P., 2002, A Monte Carlo simulation of the cosmic rays interactions with the near Earth environment, Perugia (Italy), Ph.D. thesis, http://ams.pg.infn.it/Tesi/tesi_zuccon.pdf.

Zuccon, P., Bertucci, B., Alpat, B.et al., Atmospheric production of energetic protons, electrons and positrons observed in near Earth orbit, Astroparticle Physics, 20, 221-234, doi:10.1016/S0927-6505(03)00160-9 